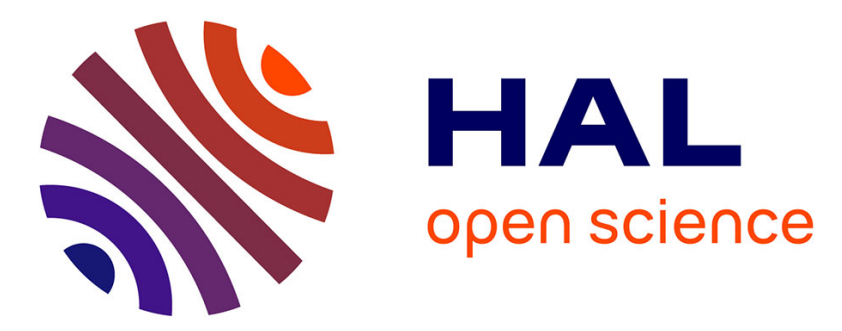

\title{
Maintaining maximum signal-to-noise ratio in uncooled vertical-cavity surface-emitting laser-based self-mixing sensors
}

\author{
Ranveer S. Matharu, Julien Perchoux, Russell Kliese, Yah Leng Lim, \\ Aleksandar D. Rakic
}

\section{To cite this version:}

Ranveer S. Matharu, Julien Perchoux, Russell Kliese, Yah Leng Lim, Aleksandar D. Rakic. Maintaining maximum signal-to-noise ratio in uncooled vertical-cavity surface-emitting laser-based self-mixing sensors. Optics Letters, 2011, 36 (18), pp.3690-3692. hal-00757555

\section{HAL Id: hal-00757555 https://hal.science/hal-00757555}

Submitted on 27 Nov 2012

HAL is a multi-disciplinary open access archive for the deposit and dissemination of scientific research documents, whether they are published or not. The documents may come from teaching and research institutions in France or abroad, or from public or private research centers.
L'archive ouverte pluridisciplinaire HAL, est destinée au dépôt et à la diffusion de documents scientifiques de niveau recherche, publiés ou non, émanant des établissements d'enseignement et de recherche français ou étrangers, des laboratoires publics ou privés. 


\title{
Maintaining maximum signal-to-noise ratio in uncooled vertical-cavity surface-emitting laser-based self-mixing sensors
}

\author{
Ranveer S. Matharu, ${ }^{1}$ Julien Perchoux, ${ }^{2,3}$ Russell Kliese, ${ }^{1}$ Yah Leng Lim, ${ }^{1}$ and Aleksandar D. Rakić ${ }^{1, *}$ \\ ${ }^{1}$ The University of Queensland, School of Information Technology and Electrical Engineering, Brisbane, QLD 4072, Australia \\ ${ }^{2}$ CNRS, LAAS, 7 Avenue du Colonel Roche, F-31077 Toulouse Cedex 4, France \\ ${ }^{3}$ Université de Toulouse, UPS, INSA, INP, ISAE, UTI, UTM, LAAS, F-31077, Toulouse Cedex 4, France \\ ${ }^{*}$ Corresponding author: rakic@itee.uq.edu.au
}

Received June 24, 2011; accepted August 9, 2011;

posted August 22, 2011 (Doc. ID 149907); published September 15, 2011

\begin{abstract}
We demonstrate a method for maintaining the maximum signal-to-noise ratio (SNR) of the signal obtained from the self-mixing sensor based on a vertical-cavity surface-emitting laser (VCSEL). It was found that the locus of the maximum SNR in the current-temperature space can be well approximated by a simple analytical model related to the temperature behavior of the VCSEL threshold current. The optimum sensor performance is achieved by tuning the laser current according to the proposed model, thus enabling the sensor to operate without temperature stabilization in a wide temperature range between $-20^{\circ} \mathrm{C}$ and $+80^{\circ} \mathrm{C}$. (C) 2011 Optical Society of America

OCIS codes: $140.2020,120.3180,140.7300$
\end{abstract}

Vertical-cavity surface-emitting lasers (VCSELs) are widely used in high-speed metropolitan and local area networks. Thanks to their advantages over in-plane lasers, VCSELs are now also making inroads into the laser sensing field.

Several VCSEL-based self-mixing (SM) sensors have been demonstrated so far [1,2]. The SM effect occurs when light reflected from a target enters the laser cavity and is added coherently to the cavity field causing gain modulation, which can be observed as the laser optical power variation and laser terminal voltage variation [3]. The importance of the ambient temperature on laser $\overline{\mathrm{SM}}$ sensors and the need for temperature stabilization in inplane and DFB lasers have been discussed earlier [ㄴ, $\underline{5}]$. Single longitudinal-mode operation in VCSELs, achieved due to the extremely short cavity, comes with the price of high temperature sensitivity, well above that for in-plane lasers [6]. The gain peak and the VCSEL cavity mode shift at different rates with temperature, leading to increasing spectral detuning and finally the well-known power rolloff [7]. The rate of the longitudinal-mode wavelength change with temperature is determined by the temperature sensitivity of the refractive index and by thermal expansion of the cavity [8]. The most successful method to counteract the thermal problems and widen the temperature operating range of VCSELs is referred to as the "gain offset design" [9]. This results in the parabolic dependence of threshold current on temperature [10]. Therefore, the thermal properties of VCSELs are strongly influencing their performance, especially when operating in an uncooled regime and with varying ambient temperatures.

In this study we investigate the interplay between the VCSEL bias conditions and the thermal effects on the signal-to-noise ratio (SNR) of the SM signal for VCSELbased SM sensors over a wide temperature range of $-40{ }^{\circ} \mathrm{C}$ to $+80^{\circ} \mathrm{C}$. We demonstrate an efficient way of maintaining optimal SNR in an SM sensor operating over a wide range of ambient temperatures without resorting to the use of an active cooling system.
Despite the high reflectivities of the DBR mirrors, VCSELs have sensitivity to optical feedback similar to that of in-plane lasers [11]. Conventional SM sensors use the photocurrent from an integrated photodiode as a source of the SM signal. We have earlier reported an alternative and significantly simpler scheme of implementing a VCSEL-based SM sensor (driven from a constant current source), which uses the variation in laser terminal voltage as a source of the SM signal [2]. While the photocurrent signal depends directly on the variations in photon density in the VCSEL cavity (optical power), the terminal voltage signal is related to a number of processes in the device. The major mechanisms leading to small changes in terminal voltage include (i) the variations in the quasi-Fermi level separation causing the junction voltage changes, due to the carrier depletion/ generation in the quantum-well active region, and (ii) the photoconduction effects in the DBR mirrors caused by the reflected beam where the generated carriers reduce the mirrors' series resistance, resulting in a decrease of the terminal voltage $[\underline{12}, 13]$. The increase in temperature leads to the increase in the amplitude of the terminal voltage signal through the increase in the laser junction voltage, the resistance of the DBR mirrors and of the separate confinement heterostructure [13]. Therefore, the magnitude of the feedback-caused voltage variations that derive from the variations in the carrier density caused by optical feedback is strongly impacted by changes in the ambient temperature.

We investigated the temperature dependence of the SM signal using the sensor in the velocimetry mode. The laser beam is scattered from a rough object moving with constant velocity and angled such that a Doppler shift is imparted on the wave reentering the laser. Through the SM process, the Doppler frequency becomes observable in the SM voltage signal [2]. The experimental setup used (shown in Fig. 1) is based on a single-mode proton-implanted VCSEL (Litrax LX-VCS-850-T101). We have measured the SNR of the SM signal for a range of laser bias currents and ambient temperatures. From this 


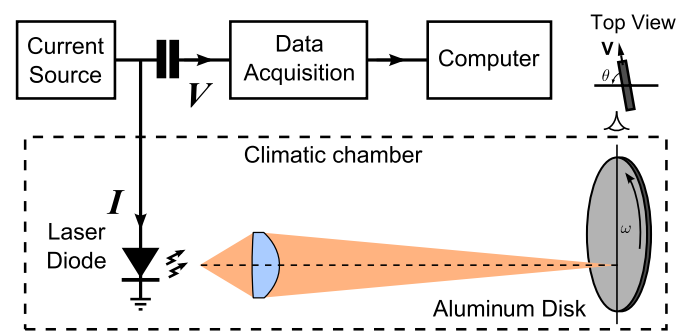

Fig. 1. (Color online) Experimental setup for the SM experiments.

set of results, we have obtained the relationship between the maximum SNR and the corresponding laser current at a given ambient temperature. The target was a sandblasted aluminum disk with a diameter of $100 \mathrm{~mm}$ driven by a DC servomotor with a 43:1 gear reduction. An optical encoder and motor controller were used to ensure that the motor rotated at a fixed angular velocity. All experiments were carried out in a climatic chamber (Climats Excal 220) to provide controlled temperatures between $-40{ }^{\circ} \mathrm{C}$ and $+80{ }^{\circ} \mathrm{C}$ in $2.5^{\circ} \mathrm{C}$ increments. After amplification, the small voltage variations across the VCSELs terminals were fed into a 12 bit data acquisition card. The incoming signal was sampled at a rate of $100 \mathrm{kHz}$ capturing 2500 sample points. The SM Doppler spectra were then obtained using the fast Fourier transform (FFT) of the acquired time-domain signal. The angle of incidence was adjusted to produce a Doppler frequency peak $\left(f_{D}\right)$ at around $10 \mathrm{kHz}$ for a beam focused $40 \mathrm{~mm}$ from the center of the disk. Signal acquisition, temperature, and current automation were carried out using a custom-built application in the LabVIEW programming environment.

Each Doppler spectrum was an ensemble average of 50 spectra obtained by applying FFT on the time-domain signal. This frequency spectrum allowed for extraction of $f_{D}$, signal peak level, and the noise floor level using software written in MATLAB. The SNR was obtained by fitting the fundamental spectral feature of the Doppler signal using the method proposed earlier [14]. Figure 2 shows a sample SM Doppler signal and the fit used to estimate the SNR. The noise floor observed can be attributed to the noise processes in the VCSEL, including the intensity noise and the junction noise. The noise floor of the electronic instrumentation is negligible, being more than $20 \mathrm{~dB}$ below the laser noise level.

The light-current (L-I) characteristic of the laser was measured for a range of temperatures from $-40{ }^{\circ} \mathrm{C}$ to $+80^{\circ} \mathrm{C}$ (Fig. 3). The differentiated L-I curves were used to determine the threshold current variation with

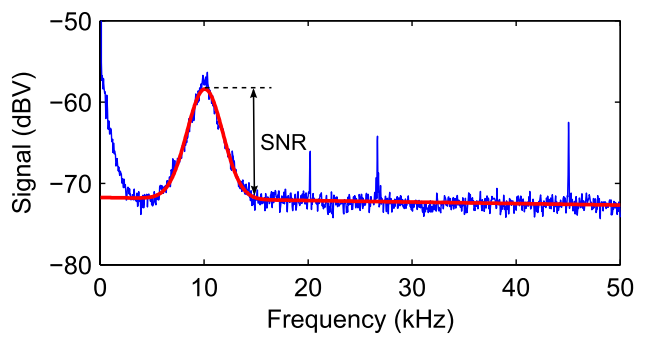

Fig. 2. (Color online) Sample SM Doppler spectrum overlaid with a fit function consisting of a Gaussian plus a constant value used to estimate the SNR.
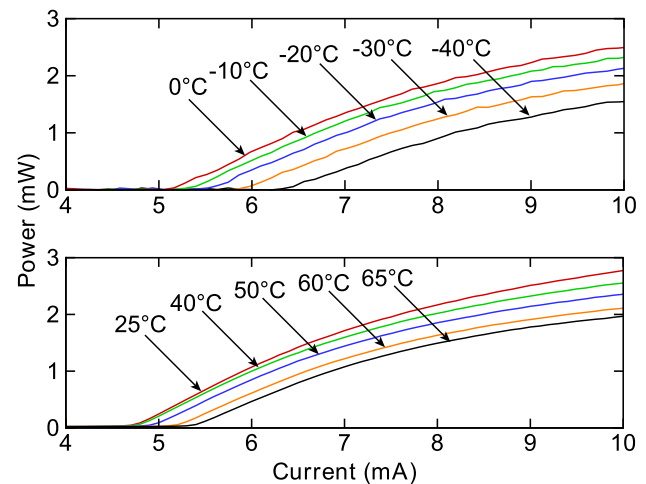

Fig. 3. (Color online) Light-current curves for the device at various temperatures showing the variation in threshold current with temperature.

temperature. The temperature dependence of the VCSEL threshold current is shown in Fig. 4, with the threshold current showing a parabolic dependence on temperature, typical for VCSELs with gain offset design $[\underline{9}, 10]$. The variation of threshold current with temperature can be well approximated by the parabolic relationship [10]

$$
I_{\text {th }}=\alpha+\beta\left(T-T_{\text {min }}\right)^{2},
$$

where $T_{\min }$ is the temperature associated with the lowest threshold current and $\alpha$ is the threshold current at $T_{\min }$. The third parameter, $\beta$, indicates the VCSEL's threshold current sensitivity to temperature change. These parameters are all device specific with $\beta$ governed by the quantum-well design and overall effective refractive index of the cavity [10]; a larger value of $\beta$ implies higher temperature sensitivity of the threshold current. At temperatures away from $T_{\min }$, the mismatch between the gain and the cavity resonant mode increases resulting in higher drive currents necessary to reach the threshold.

The model parameters extracted for the VCSEL used here are $\alpha=4.74 \mathrm{~mA}, \quad \beta=3.36 \times 10^{-4} \mathrm{~mA} /{ }^{\circ} \mathrm{C}^{2}$, and $T_{\text {min }}=28.5^{\circ} \mathrm{C}$.

The SNR of the SM signal is of interest for all SM sensors, and it has been known to depend on both the temperature and the bias current [15]. Figure 4 shows the SNR of the SM signal as a function of the laser current

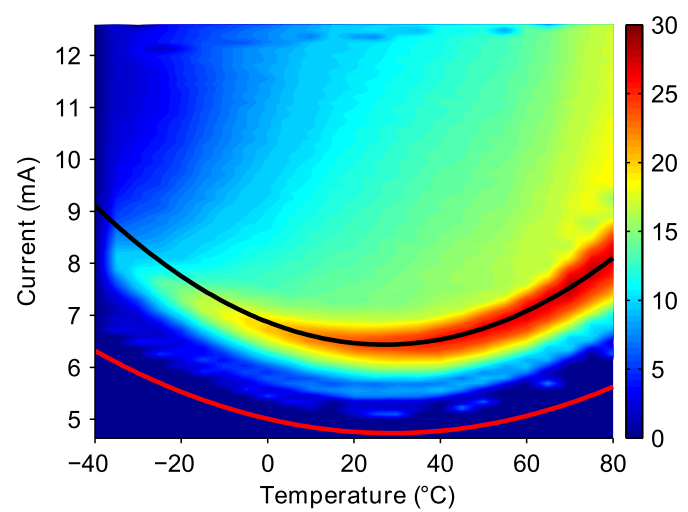

Fig. 4. (Color online) SNR of the SM Doppler signal as a function of temperature and current. The red solid (lowest) curve represents the temperature dependence of the threshold current, and the black solid (highest) curve is a locus of the maximum SNR for the VCSEL operating in a single-mode regime. 
and the laser heat-sink temperature. The SNR was up to $30 \mathrm{~dB}$ in the range of investigated temperatures. The experimental data show that the maximum SNR is obtained for currents close to the threshold, as suggested earlier for in-plane lasers [15]. As the current further increases above the threshold, the amplitude of the Doppler signal decreases for any given temperature due to the combined effects of increased injection level on the laser series resistance and the separation between the quasi-Fermi levels [12]. The increase in both the signal level and the SNR with temperature can be inferred from the current voltage characteristic of a multiple quantum-well VCSEL [13].

We propose here that the locus of the maximum SNR can be described by the behavioral model resembling that of the threshold current temperature dependence and that the current required for the maximum SNR $\left(I_{\mathrm{SNR}}\right)$ as a function of the laser heat-sink temperature can be approximated by

$$
I_{\mathrm{SNR}}=\alpha_{\mathrm{SNR}}+\beta_{\mathrm{SNR}}\left(T-T_{\mathrm{SNR}}\right)^{2},
$$

where $\alpha_{\mathrm{SNR}}$ is the current for the maximum SNR at the optimal temperature, $T_{\mathrm{SNR}}$, and the parameter $\beta_{\mathrm{SNR}}$ describes the temperature sensitivity of the SM signal's SNR for this particular VCSEL. The parameter values obtained by fitting Eq. (2) to the experimental data [solid black curve in Fig. 4] are $\alpha_{\mathrm{SNR}}=6.44 \mathrm{~mA}, \beta_{\mathrm{SNR}}=5.95 \times$ $10^{-4} \mathrm{~mA} /{ }^{\circ} \mathrm{C}^{2}$ and $T_{\mathrm{SNR}}=27.1^{\circ} \mathrm{C}$, a value almost identical to that of $T_{\min }$. The higher value of the $\beta_{\mathrm{SNR}}$ when compared to $\beta$ shows the need for increased current above threshold at temperatures far from the design temperature $T_{\min }$. This model for maximum SNR can be used to tune the laser bias current to maintain the maximum SNR for the SM signal over a wide temperature range using close-loop control, and it offers considerable power savings over Peltier-effect cooling.

This study investigated the current and temperature dependence of SM signal SNR in a VCSEL-based sensor and proposed a new method for maintaining the maximum SNR over a wide range of operating temperature. The relationship between the laser current for optimal SNR and the heat-sink temperature closely resembles the parabolic relationship between the threshold current and the ambient temperature. This suggests that the maximum SNR of the signal from a VCSEL-based SM sensor can be related to the ambient temperature by the simple parabolic model based on the model for the temperature behavior of the VCSEL threshold current. This approach enables one to maintain the SNR of the sensor between $20-30 \mathrm{~dB}$ in a wide range of ambient temperatures between -20 and $+80^{\circ} \mathrm{C}$ by tuning the laser current according to the proposed model and operate the sensor without temperature stabilization, making it attractive for low-power and mobile applications.

This research was supported under the Australian Research Council's Discovery Projects funding scheme (DP0988072) and the Commonwealth of Australia International Science Linkages program (FR090026).

\section{References}

1. J. R. Tucker, A. D. Rakic, C. J. O'Brien, and A. V. Zvyagin, Appl. Opt. 46, 611 (2007).

2. Y. L. Lim, R. Kliese, K. Bertling, K. Tanimizu, P. A. Jacobs, and A. D. Rakic, Opt. Express 18, 11720 (2010).

3. T. Bosch, C. Bes, L. Scalise, and G. Plantier, in Encyclopedia of Sensors, Vol. X, C. A. Grimes, E. C. Dickey, and M. V. Pishko, eds. (American Scientific, 2006), p. 1.

4. S. Donati, L. Falzoni, and S. Merlo, IEEE Trans. Instrum. Meas. 45, 942 (1996).

5. L. Scalise, W. Steenbergen, and F. de Mul, Appl. Opt. 40, 4608 (2001).

6. J. Piprek, H. Wenzel, and G. Sztefka, IEEE Photon. Technol. Lett. 6, 139 (1994).

7. P. Mena, J. Morikuni, S.-M. Kang, A. Harton, and K. Wyatt, J. Lightwave Technol. 17, 865 (1999).

8. J. J. Dudley, D. L. Crawford, and J. E. Bowers, IEEE Photon. Technol. Lett. 4, 311 (1992).

9. D. Young, J. Scott, F. Peters, M. Peters, M. Majewski, B. Thibeault, S. Corzine, and L. Coldren, IEEE J. Quantum Electron. 29, 2013 (1993).

10. C. Chen, P. Leisher, A. Allerman, K. Geib, and K. Choquette, IEEE J. Quantum Electron. 42, 1078 (2006).

11. K. Petermann, IEEE J. Sel. Top. Quantum Electron. 1, 480 (1995).

12. Y. Mitsuhashi, J. Shimada, and S. Mitsutsuka, IEEE J. Quantum Electron. 17, 1216 (1981).

13. G. Taylor and Q. Yang, IEEE J. Quantum Electron. 32, 1441 (1996).

14. R. Kliese, Y. L. Lim, T. Bosch, and A. D. Rakić, Opt. Lett. 35, 814 (2010).

15. K. B. Rochford and A. H. Rose, Opt. Lett. 20, 2105 (1995). 